\title{
Spin colour centres in SiC as a material platform for sensing and information processing at ambient conditions
}

\author{
Andrey Anisimov ${ }^{1, *}$, Victor Soltamov ${ }^{1,2}$, Pavel Baranov ${ }^{1}$, Georgy Astakhov ${ }^{2,3}$ and Vladimir \\ Dyakonov $^{2}$ \\ ${ }^{1}$ A.F. Ioffe Institute RAS, Politekhnicheskaya 26, 194021, Saint Petersburg, Russia \\ ${ }^{2}$ Experimental Physics VI, Julius-Maximilians University of Wuerzburg, 97074 Wuerzburg, Germany \\ ${ }^{3}$ Helmholtz-Zentrum Dresden-Rossendorf, Institute of Ion Beam Physics and Materials Research, \\ 01328 Dresden, Germany
}

\begin{abstract}
Atomic-scale colour centres in bulk and nanocrystalline $\mathrm{SiC}$ are promising systems for quantum photonics compatible with fiber optics, quantum information processing and sensing at ambient conditions. Colour centres which acts as stable single photon sources in $\mathrm{SiC}$ can be key elements for quantum photonics and communications. It has been shown that there are at least two families of colour centres in $\mathrm{SiC}$ with $\mathrm{S}=1$ and $\mathrm{S}$ $=3 / 2$, which have the property of optical alignment of the spin levels even at room temperature and above. The spin state can be initialized, manipulated and readout by means of optically detected magnetic resonance (ODMR), level anticrossing and cross-relaxation. Recently, we observed the effects of "hole burning" in the ODMR spectra, which made it possible to narrow the ODMR line by approximately an order of magnitude, which substantially increases the possibilities of technological applications of spin centres.
\end{abstract}

The unique quantum properties of the NV centre in diamond [1,2] have motivated efforts to find defects with similar properties in silicon carbide ( $\mathrm{SiC}$ ), which can extend the functionality of such systems. Atomic-scale colour centres in bulk and nanocrystalline SiC are promising for quantum information processing, photonics compatible with fiber optics and sensing at ambient conditions. Their spin state can be initialized, manipulated and readout by means of optically detected magnetic resonance (ODMR), level anticrossing (LAC), cross-relaxation (CR) [4] and hole-burning radio-frequency spectrum analysis [3]. It has been shown that there are at least two families of colour centres in $\mathrm{SiC}$ with $\mathrm{S}=1$ and $\mathrm{S}=3 / 2$, which have the property of optical alignment of the spin levels and allow a spin manipulation. For the $\mathrm{S}=3 / 2$ family, the ground state and the excited state were demonstrated to have spin $\mathrm{S}=3 / 2$ and a population inversion in the ground state can be generated using optical pumping, leading to stimulated microwave emission even at room temperature and above. By controlling the neutron irradiation fluence, the colour centres concentration can be varied over several orders of magnitude down to a single defect level.

\footnotetext{
*Corresponding author: aan0100@gmail.com
} 
Furthermore, these atomic-scale spin colour centres can be also attractive for local or environment sensing. Several, separately addressable spin-3/2 centres (spin qudits) have been identified in the same crystal for each polytype (4H-, $6 \mathrm{H}-$ and $15 \mathrm{R}-\mathrm{SiC}$ ) which can be used either for magnetic field or temperature sensing. Some of these spin colour centres are characterized by nearly temperature independent zero-field splitting, making these centres very attractive for vector magnetometry. Contrarily, the zero-field splitting of the centres in the excited state exhibits a giant thermal shift, which can be used for thermometry applications. $\mathrm{SiC}$ is taking on a new role as a flexible and practical platform for harnessing the new quantum technologies, it is a technologically material, used in various devices (LED, MOSFETS, MEMS).

Here, we apply a two-frequency to excite and image multiple qudit modes in a SiC spin ensemble under ambient conditions. Strikingly, their spectral width is about one order of magnitude narrower than the inhomogeneous broadening of the corresponding spin resonance [3].

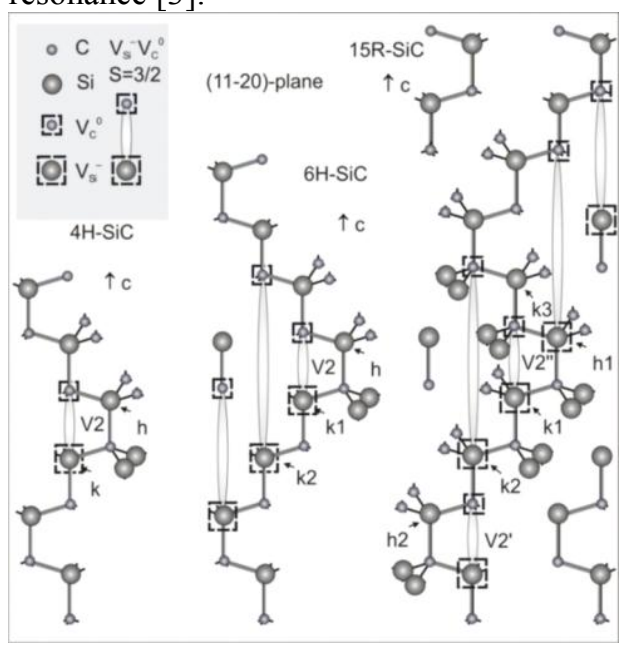

Fig. 1. Models showing possible configurations familie of spin colour centres in $4 \mathrm{H}-\mathrm{SiC}, 6 \mathrm{H}-$ $\mathrm{SiC}$ and 15R-SiC lattice in (11-20) plane with $\mathrm{S}=3 / 2$.

corresponding spin resonance.

We have presented a review of optical and spin properties of colour centres in different polytypes of $\mathrm{SiC}$, which were studied two-radiofrequency hole-burning spectrum analysis. This study will allow SiC is taking on a new role as a flexible and practical platform for harnessing the new quantum technologies.

This work was supported by the by the RFBR under Grant № 16-02-00877-a; by the RSF № 16-4201098 and V.A.S. acknowledges support through a sponsorship provided by the Alexander von Humboldt $(\mathrm{AvH})$ foundation fellowship.

\section{References}

1. A. Gruber et al., Science 276, 2012 (1997)

2. P. G. Baranov et. al., Magnetic Resonance of Semiconductors and Their Nanostructures: Basic and Advanced Applications (Springer, 2017)

3. V. A. Soltamov et al., arXiv:1807.10383

4. A. N. Anisimov et al., AIP Advances 8, 085304 (2018) 